\title{
Bisphenol AF and Bisphenol B Exert Higher Estrogenic Effects than Bisphenol A via G Protein-Coupled Estrogen Receptor Pathway
}

Lin-Ying Cao, ${ }^{\dagger, \dagger}$ Xiao-Min Ren, ${ }^{*}{ }^{\dagger}$ Chuan-Hai Li, ${ }^{\dagger \dagger}$ Jing Zhang, ${ }^{\dagger}$ Wei-Ping Qin, ${ }^{\dagger \dagger}$ Yu Yang, ${ }^{\dagger}$ Bin Wan, and Liang-Hong Guo*,,$+\neq 0$

${ }^{\dagger}$ State Key Laboratory of Environmental Chemistry and Eco-toxicology, Research Center for Eco-environmental Sciences, Chinese
Academy of Sciences, 18 Shuangqing Road, Beijing 100085, P. R. China

\#University of Chinese Academy of Sciences, Beijing 100039, P. R China

Supporting Information

ABSTRACT: Numerous studies have indicated estrogenic disruption effects of bisphenol A (BPA) analogues. Previous mechanistic studies were mainly focused on their genomic activities on nuclear estrogen receptor pathway. However, their nongenomic effects through $G$ protein-coupled estrogen receptor (GPER) pathway remain poorly understood. Here, using a SKBR3 cell-based fluorescence competitive binding assay, we found six BPA analogues bound to GPER directly, with bisphenol AF (BPAF) and bisphenol B (BPB) displaying

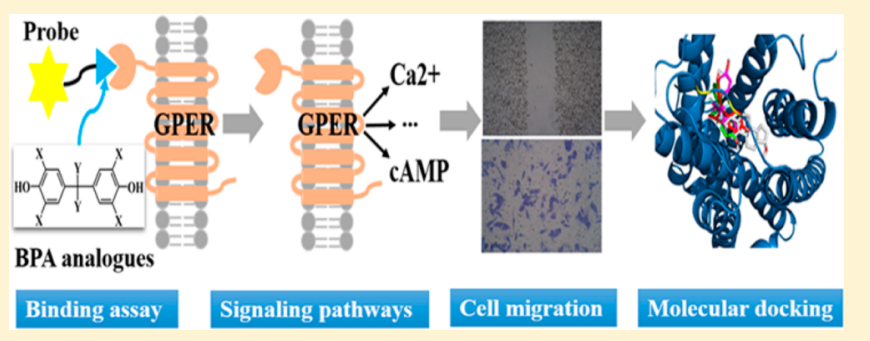
much higher ( 9-fold) binding affinity than BPA. Molecular docking also demonstrated the binding of these BPA analogues to GPER. By measuring calcium mobilization and cAMP production in SKBR3 cells, we found the binding of these BPA analogues to GPER lead to the activation of subsequent signaling pathways. Consistent with the binding results, BPAF and BPB presented higher agonistic activity than BPA with the lowest effective concentration (LOEC) of $10 \mathrm{nM}$. Moreover, based on the results of Boyden chamber and wound-healing assays, BPAF and BPB displayed higher activity in promoting GPER mediated SKBR3 cell migration than BPA with the LOEC of 100 nM. Overall, we found two BPA analogues BPAF and BPB could exert higher estrogenic effects than BPA via GPER pathway at nanomolar concentrations.

\section{INTRODUCTION}

Bisphenol A (BPA) is a ubiquitous environment pollutant detected in the environment, wildlife, and humans. ${ }^{1}$ Exposure of BPA is suspected to affect a variety of physiological functions and to be associated with many human diseases. ${ }^{2-6}$ Facing the increased concern over the safety of BPA, some BPA analogues have been developed as alternatives of $\mathrm{BPA}^{7}$ For example, bisphenol S (BPS) and bisphenol F (BPF) have been used as BPA substitutes in the production of epoxy resins used as food contact material. ${ }^{8}$ Bisphenol AF (BPAF) is wildly used in polycarbonate copolymers in high-temperature composites, electronic materials, and gas-permeable membranes. Bisphenol $\mathrm{B}$ (BPB) is utilized in the manufacture of resins and plastics. Besides, some halogenated derivatives of BPA such as tetrabromobisphenol A (TBBPA) and tetrachlorobisphenol A (TCBPA) are used as flame retardants. ${ }^{7}$ Now, these BPA analogues have become emerging environmental pollutants with some analogues occurring at similar or even higher concentrations than that of $\mathrm{BPA} .^{7,9}$ For example, the concentrations of BPS $(4.92 \mathrm{ng} / \mathrm{mL})$ and BPF $(2.16 \mathrm{ng} / \mathrm{mL})$ in urines from a population in Saudi Arabia surpassed that of BPA $(2.01 \mathrm{ng} / \mathrm{mL}){ }^{7}$ The concentration of BPB $(5.15 \mathrm{ng} / \mathrm{mL})$ was also higher than that of BPA $(2.91 \mathrm{ng} / \mathrm{mL})$ in serum from healthy and endometriotic women in Italy. ${ }^{9}$
The estrogenic effects of bisphenols especially BPA have been widely investigated previously. ${ }^{10,11}$ Studies on the molecular mechanisms have revealed that BPA could exert genomic estrogenic effects via binding to and activating the nuclear estrogen receptor (ER). In recent years, there is growing evidence showing that BPA could also exert nongenomic estrogenic effects by activating $G$ protein-coupled estrogen receptor (GPER) pathway at low concentrations. ${ }^{12-14}$ For example, nanomolar level BPA stimulated the proliferation of mouse spermatogonial cells, ${ }^{15}$ human seminoma cells ${ }^{16}$ and testicular seminoma cells ${ }^{17}$ through the activation of GPER. Furthermore, Tomas and Dong demonstrated BPA could bind to human GPER directly with relative binding affinity (RBA) of $2.8 \%$ compared to $17 \beta$-estradiol (E2). ${ }^{18}$ Besides, Fitzgerald et al. showed BPA could also bind to zebrafish GPER with RBA of $15.8 \%$ compared to E2. ${ }^{19}$

The structural similarity of BPA analogues implies the potential similar estrogenic effects as BPA. Actually, various estrogenic effects of BPA analogues have been observed. For example, exposure of 8,40 , and $100 \mathrm{mg} / \mathrm{kg} \mathrm{BPAF}$ to rats

Received: July 1, 2017

Revised: August 26, 2017

Accepted: August 31, 2017

Published: August 31, 2017 
resulted in uterotrophic significantly. ${ }^{20}$ Exposure of $0.5,1$, and $1.5 \mathrm{mg} / \mathrm{L} \mathrm{BPAF}$ to zebrafish promoted the expression of vitellogenin. ${ }^{21}$ Besides, several BPA analogues such as BPS, BPF and BPAF have been found to activate the expression of estrogenic markers in the brain of developing zebrafish. ${ }^{22}$ By systematic review and comparison of the estrogen hormonal activity of bisphenol A substitutes, Rochester and Bolden showed BPS and BPF had similar activity as BPA in the same order of magnitude of potency. ${ }^{8}$ Similar to earlier studies of $\mathrm{BPA}$, previous mechanistic studies of BPA analogues also focused on their effects on ER pathway. For example, BPAF, $\mathrm{BPB}, \mathrm{BPF}$, and BPS were found to have similar or even higher binding affinity and activity to ER than BPA. ${ }^{8,23,24}$ Since the previous studies have proved BPA could exert estrogenic effects at low concentrations through the GPER pathway, it is very essential to clarify whether the BPA analogues can also exert similar effects.

Actually, several in vitro studies have shown that some BPA analogues might exert nongenomic estrogenic effects via GPER. For example, by silencing the GPER gene, Li et al. revealed GPER was involved in the endogenous transcription of estrogen responsive genes in human breast cancer cells induced by $100 \mathrm{nM} \mathrm{BPAF} .^{23}$ By using GPER selective inhibitor G15, Vinas and Watson found GPER was involved in the disruption effects of BPS $\left(10^{-14} \mathrm{M}\right)$ on E2-induced membrane-initiated cell signaling. ${ }^{26}$ Similarly, by using G15, Fitzgerald et al. found nanomolar level TCBPA and TBBPA could inhibit oocytes maturation of zebrafish through GPER pathway. ${ }^{19}$ These limited studies have showed GPER pathway might be involved in the estrogenic effects of BPA analogues. However, direct binding of BPA analogues to GPER remain poorly understood, and there is still a lack of comparative study for their effects on GPER pathway, which is essential for risk assessment and development of safe BPA substitutes.

In the present study, we aimed to investigate whether BPA analogues could also exert nongenomic estrogenic effects via GPER pathway as BPA did. Direct binding of BPA analogues to GPER were investigated by SKBR3 cell-based fluorescence competitive binding assay and molecular docking. Furthermore, the activities of BPA analogues on GPER mediated signaling pathway and SKBR3 cell migration function were also examined.

\section{EXPERIMENTAL SECTION}

Chemicals. Seven bisphenols including BPA, BPS, BPF, $\mathrm{BPB}, \mathrm{BPAF}, \mathrm{TCBPA}$, and TBBPA were purchased from J\&K Scientific Ltd. (Beijing, China). All the bisphenols had a purity of $98 \%$ or higher and were solubilized in dimethyl sulfoxide (DMSO) to make stock solutions with a concentration of 50 mM. E2 (98\%) was purchased from Sigma-Aldrich (St. Louis, MO). G15 (98\%) was purchased from Cayman Chemical Company (Ann Arbor, MI). Fura-2 AM calcium indicator and cAMP-screen system were purchased from Invitrogen (Carlsbad, CA). The structures and CAS numbers of E2 and bisphenols are shown in Supporting Information (SI) Figure S1.

Cell Treatments. SKBR3 breast cancer cells and Human HEK 293 embryonal kidney cells were purchased from the American Type Culture Collection (Manassas, VA). The HEK 293 cells were cultured in Dulbecco's modified Eagle's medium (DMEM) (Invitrogen) supplemented with $10 \%$ fetal bovine serum (FBS, HyClone, Waltham, MA), $100 \mathrm{U} / \mathrm{mL}$ penicillin (Invitrogen) and $100 \mu \mathrm{g} / \mathrm{mL}$ streptomycin (Invitrogen) at 37
${ }^{\circ} \mathrm{C}$ in a humidified $5 \% \mathrm{CO}_{2}$ atmosphere. The SKBR3 cells were maintained in phenol red-free Roswell Park Memorial Institute (RPMI) 1640 (Invitrogen) with $10 \% \mathrm{FBS}, 100 \mathrm{U} / \mathrm{mL}$ penicillin and $100 \mu \mathrm{g} / \mathrm{mL}$ streptomycin at $37{ }^{\circ} \mathrm{C}$ in a humidified $5 \% \mathrm{CO}_{2}$ atmosphere. The cells in all the experiments were from homogeneous cell suspension and the vehicle control group (Ctrl, 0.1\% DMSO) was tested concurrently. The experimental conditions were the same for different groups in all the experiments.

SKBR3 Cell-Based Fluorescence Competitive Binding Assay. The binding affinities of the seven bisphenols to GPER were detected by SKBR3 $\left(\mathrm{GPER}^{+}, \mathrm{ER}^{-}\right)$cell-based fluorescence competitive binding assay. The establishment and validation of the method were described in SI in detail (Figure S2). In brief, $100 \mu \mathrm{L}$ harvested SKBR3 cells $\left(\sim 1 \times 10^{5}\right.$ cells), $50 \mathrm{nM} \mathrm{E2-F} \mathrm{(a}$ fluorescence probe synthesized by conjugating E2 with fluorescein through a linker, the structure is shown in SI Figure S1), and tested compounds were mixed in phosphatebuffered saline buffer $(137 \mathrm{mM} \mathrm{NaCl}, 2.7 \mathrm{mM} \mathrm{KCl}, 10 \mathrm{mM}$ $\mathrm{Na}_{2} \mathrm{HPO}_{4}$, and $\left.1.8 \mathrm{mM} \mathrm{KH}_{2} \mathrm{PO}_{4}, \mathrm{pH} 7.4\right)$ in a total volume of $500 \mu \mathrm{L}$ and incubated for $10 \mathrm{~min}$ at $37{ }^{\circ} \mathrm{C}$. Then the FITC fluorescence intensity of cell samples was measured on a NovoCyte flow cytometer (ACEA Biosciences, CA). The displacement of E2-F binding by a competitor was expressed as a percentage of the maximum specific binding to obtain the competition curve. The competition curves were fitted with a sigmoidal equation using SigmaPlot 12.0 (Systat Software Inc., San Jose, CA) to obtain an $\mathrm{IC}_{50}$ value (the concentration of a ligand required to displace $50 \%$ of the probe from the receptor). The tested bisphenols had no effect on the nonspecific binding of E2-F to the control HEK293 cells $\left(\mathrm{GPER}^{-}, \mathrm{ER}^{-}\right)$(SI Figure S3).

Intracellular Calcium Mobilization and cAMP Assay. Calcium mobilization was detected by using a fluorescence indicator fura 2-AM (Invitrogen). After serum starvation for 24 h, SKBR3 cells $\left(5 \times 10^{6}\right)$ were treated with $5 \mu \mathrm{M}$ fura- $2 \mathrm{AM}$ in the presence of $0.05 \%$ pluronic acid at $37^{\circ} \mathrm{C}$ for $30 \mathrm{~min}$. Then, the cells were washed with phenol red-free RPMI 1640 three times and incubated for another $30 \mathrm{~min}$. The cells were harvested by trypsin-EDTA, washed with phenol red-free RPMI 1640 , resuspended in phenol red-free RPMI 1640 at a density of $1 \times 10^{7}$ cells $/ \mathrm{mL}$ and kept on ice until assay. We determined calcium mobilization ratiometrically using $\lambda_{\mathrm{ex}} 340 / 380 \mathrm{~nm}$ and $\lambda_{\mathrm{em}} 510 \mathrm{~nm}$ in a Horiba Fluoromax-4 spectrofluorometer (Edison, NJ). The cAMP production was determined using the cAMP-Screen Direct System (Invitrogen). SKBR3 cells were seeded in precoated 96-well microplates provided by Invitrogen $\left(2 \times 10^{4}\right.$ cells/well $)$ and incubated for $24 \mathrm{~h}$. Then, cells were serum starved in phenol red-free RPMI 1640 for another $24 \mathrm{~h}$. After that, the cells were pretreated with $1 \mathrm{mM} 3-$ isobutyl-1-methylxanthon for $30 \mathrm{~min}$ before added with a compound and incubated for another $30 \mathrm{~min}$. Finally, the cAMP concentrations were quantified according to the manufacturer's instructions.

Cell Migration Assay. SKBR3 cell migration was determined by Boyden chamber and wound-healing assays. Boyden chamber assay was performed in 24-well plates containing cell culture inserts with $8 \mu \mathrm{m}$ pore size (Corning, New York). Wound-healing assay was carried out in 24-well microplates (Corning). The detailed procedures for the two assays are provided in the SI. The concentrations of bisphenols used in cell migration assay were noncytotoxic detected by WST-1 assay (SI Figure S4). 

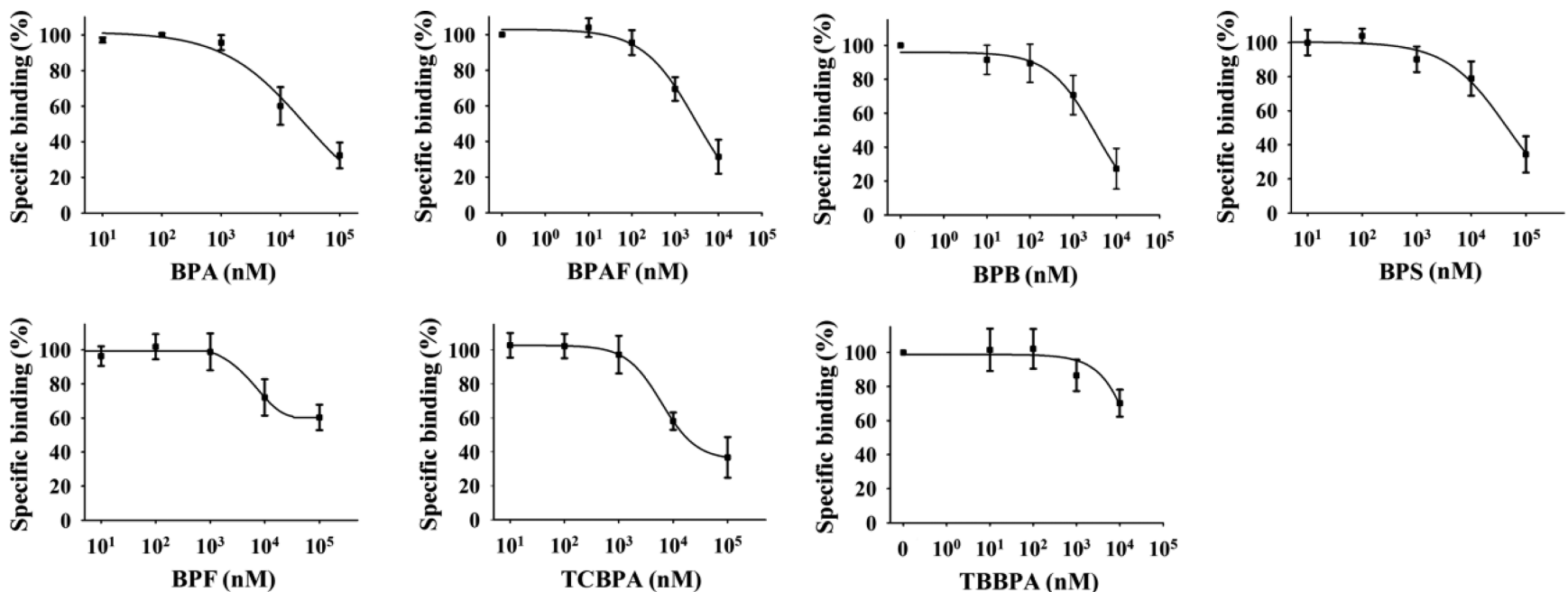

Figure 1. Determination of the binding affinities of seven bisphenols with GPER by SKBR3 cell-based fluorescence competitive binding assay.

Table 1. Results of the Binding Potency and Molecular Docking Analysis of Compounds with GPER ${ }^{a}$

\begin{tabular}{|c|c|c|c|c|c|}
\hline compounds & $\mathrm{IC}_{50}(\mu \mathrm{M})$ & RBA\% (GPER) & $\mathrm{RBA} \%(\mathrm{ER} \alpha)$ & $\Delta G(\mathrm{~kJ} / \mathrm{mol})$ & hydrogen bond \\
\hline${ }^{b} \mathrm{E} 2$ & $0.3 \pm 0.1$ & 100.0 & 100.0 & -19.4 & Asn276 \\
\hline BPAF & $3.0 \pm 0.4$ & 9.7 & $0.12,{ }^{35} 1.64^{37}$ & -16.7 & $\mathrm{G} \ln 138$ \\
\hline ВРВ & $3.3 \pm 0.6$ & 8.8 & $0.086,{ }^{36} 0.358^{37}$ & -15.5 & Cys207 \\
\hline BPA & $25.3 \pm 7.2$ & 1.1 & $0.008-0.085^{35-38}$ & -14.1 & Cys207 \\
\hline TCBPA & $25.3 \pm 10.2$ & 1.1 & $0.004^{36}$ & -13.4 & \\
\hline BPS & $45.2 \pm 9.1$ & 0.6 & $0.0009^{36}$ & -10.1 & Phe206 \\
\hline BPF & $>100.0$ & NA & $\mathrm{NA},{ }^{37} 0.0009^{36}$ & -4.8 & Phe206 \\
\hline TBBPA & $>100.0$ & NA & $\mathrm{NA}^{38}$ & -3.4 & \\
\hline
\end{tabular}

${ }^{a} \mathrm{RBA}$ (relative binding affinity): ratio between $\mathrm{IC}_{50}$ of $\mathrm{E} 2$ and compounds. The RBA values for $\mathrm{ER} \alpha$ are obtained from literatures. NA, not achieved. ${ }^{b} \mathrm{E} 2$ : the results for $\mathrm{E} 2$ are cited from our previous work (unpublished data).

Molecular Docking. The process of GPER homology modeling, protein structure and small compounds optimization were the same as described in detail in our previous work (unpublished data). The optimized compounds were docked to GPER using Autodock Vina v1.1.2 (San Francisco, CA). The docking process and ligand-protein complex analysis were the same as described in our previous work (unpublished data). The detailed procedures for homology modeling and molecular docking are provided in the SI.

Statistical Analysis. Results are expressed as mean $\pm \mathrm{SD}$ ( $n=3$, SD means the standard deviation of three measurements). The statistical analysis was performed using SPSS statistics software (Chicago, IL). The normality and homogeneity of variance of all data were analyzed by one-way analysis of variance (ANOVA) and then the multiple comparisons for differences in means was performed by Bonferroni's post hoc test. A $p$-value $<0.05$ was considered statistically significant.

\section{RESULTS AND DISCUSSION}

BPAF and BPB Showed Stronger Binding Affinity to GPER than BPA. The binding affinities of seven bisphenols including six BPA analogues as well as BPA to GPER were determined by SKBR3 cell-based fluorescence competitive binding assay. With the site-specific E2-F probe used, the binding affinities of these bisphenols were determined at the E2-binding pocket of GPER specifically. The competition binding curves are shown in Figure 1. From the competition curves, $\mathrm{IC}_{50}$ and RBA values were calculated and listed in Table 1. Based on the competitive binding results, we found all of the tested bisphenols could bind to GPER directly but with different affinity. For BPA, it bound to GPER with a RBA of $1.1 \%$ compared to E2 (Table 1 and Figure 1), which is similar to a previous study showing that BPA had a RBA of $2.8 \%$ with GPER determined by $\left[{ }^{3} \mathrm{H}\right] \mathrm{E} 2$ displacement assay. ${ }^{18}$ For the six $\mathrm{BPA}$ analogues, they bound to GPER with RBA values ranging from not achieved (NA) to 9.7\% (Table 1). Among them, $\mathrm{BPAF}$ and $\mathrm{BPB}$ even presented much stronger binding affinity ( 9-fold) than BPA, with RBA of $9.7 \%$ and $8.8 \%$ respectively (Table 1). TCBPA and BPS showed nearly comparable affinity with BPA (with RBA of $1.1 \%$ and $0.6 \%$ respectively). TBBPA and BPF exhibited too weak affinity to obtain their $\mathrm{IC}_{50}$. Here, we provided the first evidence that, like BPA, the BPA analogues could also bind to human GPER directly. BPAF and $\mathrm{BPB}$ even had much stronger binding affinity to GPER than BPA.

BPAF and BPB Docked with GPER More Effectively than BPA. We also used the molecular docking analysis to study the interactions of BPA analogues with GPER. Seven bisphenols were docked into the ligand binding pocket of GPER developed by homology modeling, whose reliability was demonstrated in our previous work (unpublished data). The structures of the docked GPER/ligand complexes are shown in Figure $2 \mathrm{~A}$ and SI Figure S5. The binding energy ( $\Delta G$ values) and hydrogen bond interactions obtained from the docking analysis are listed in Table 1. For AutoDock vina, the ligand with lower $\Delta G$ value has higher binding affinity for the receptor. As shown in Table 1 , the $\Delta G$ values of the seven tested bisphenols $(-3.4$ to $-16.7 \mathrm{kj} / \mathrm{mol})$ were much higher 

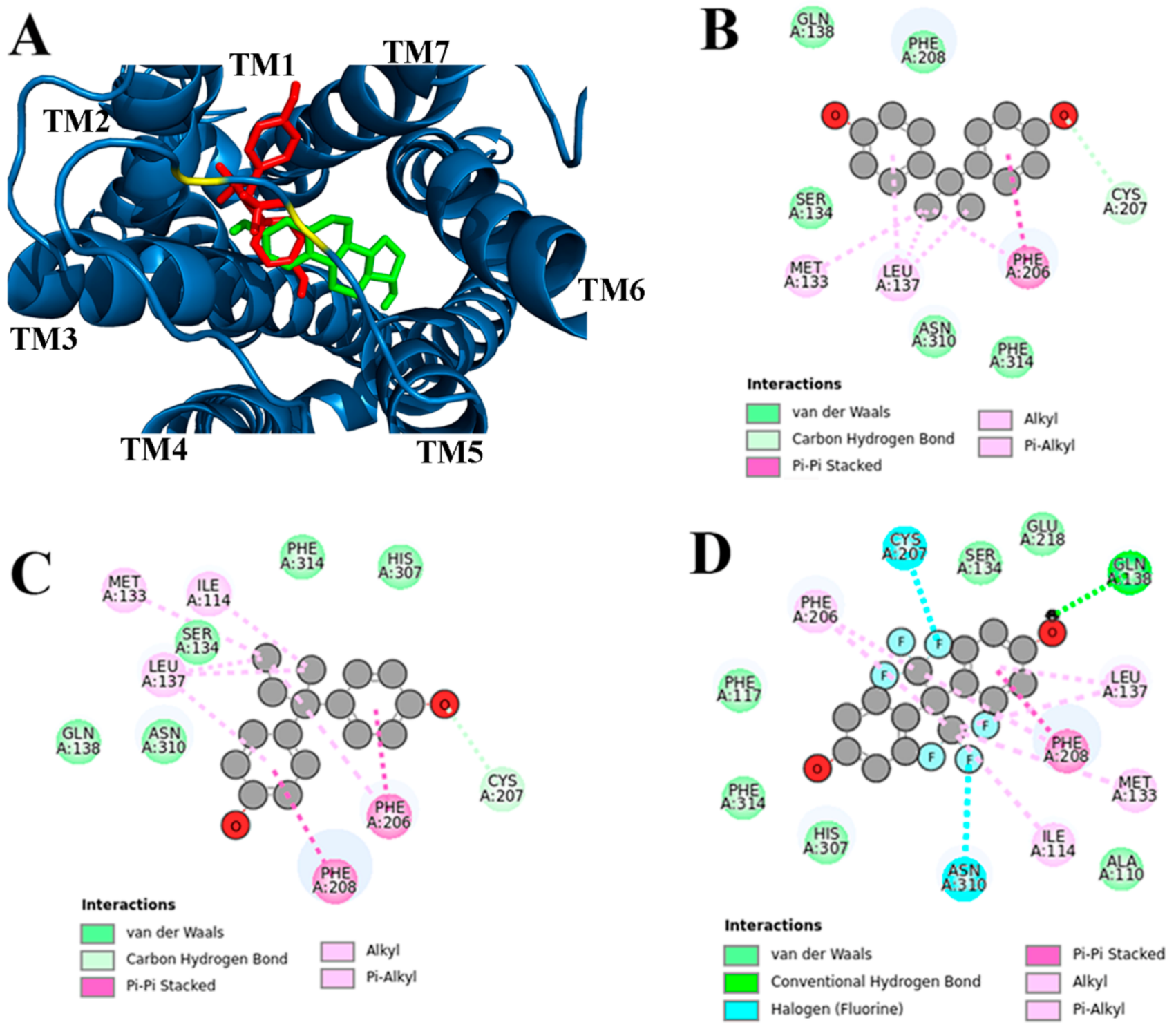

Figure 2. Docking of E2 and bisphenols into the ligand-binding pocket of GPER. (A) Overlay of the binding pose of E2 (green) and BPAF (red). Ligands are shown as sticks and GPER as blue ribbons. The helixes were labeled from TM1 to TM7. (B)-(D) The interactions of BPA, BPB, and BPAF with GPER respectively.

than that of E2 $(-19.4 \mathrm{kj} / \mathrm{mol})$, predicting that they do not bind to GPER as strongly as E2. Moreover, the binding energies of BPAF $(-16.7 \mathrm{kj} / \mathrm{mol})$ and $\mathrm{BPB}(-15.5 \mathrm{kj} / \mathrm{mol})$ were considerably lower than that of other bisphenols including BPA $(-14.1 \mathrm{kj} / \mathrm{mol})$, which suggests that BPAF and BPB would bind more preferable to GPER than the other five bisphenols including BPA. This in silico binding energy ranking order agrees well with the order of binding affinity obtained by competitive binding assay (Table 1).

BPAF and BPB Exhibited Stronger Agonistic Activity to GPER than BPA. Activation of GPER has been reported to change the subsequent signaling pathways in cells, such as triggering the rapid calcium mobilization and producing cAMP. $^{18,27,28}$ In order to evaluate the activities of the seven bisphenols on GPER, we determined their effects on the calcium mobilization and cAMP accumulation in SKBR3 cells at $1 \mu \mathrm{M}$. For BPA, it stimulated both intracellular calcium mobilization and cAMP production of SKBR3 cells (Figure 3A and Figure $4 \mathrm{~A}$ ). However, when pretreatment with $10 \mu \mathrm{M}$ G15, the effects were mostly inhibited (Figure 4A and Figure 5A). Our results are in accordance with previous studies showing the GPER agonistic activity of BPA determined by cAMP production $^{18}$ and ERK phosphorylation assays. ${ }^{14,29}$ For the six BPA analogues, we found they also evoked the increase of intracellular calcium mobilization and induced the cAMP production in SKBR3 cells (Figure 3A and Figure 4A) except $\mathrm{BPF}$ in CAMP production assay. Besides, these effects were also inhibited by $10 \mu \mathrm{M}$ G15 with the exception of BPS in cAMP production assay (Figure $3 \mathrm{~A}$ and Figure $4 \mathrm{~A}$ ). The above results suggest that, like BPA, the six BPA analogues also had agonistic activity to GPER. Currently, the information about the effect of BPA analogues on GPER is very limit. As far as we know, there were only two previous studies suggesting that BPAF might induce endogenous transcription by GPER pathway ${ }^{25}$ and BPS might disrupt E2-induced membrane-initiated cell signaling via GPER. ${ }^{26}$ Here, we found for the first time that BPA analogues could trigger the intracellular calcium mobilization and cAMP production of SKBR3 cells via GPER activation. 

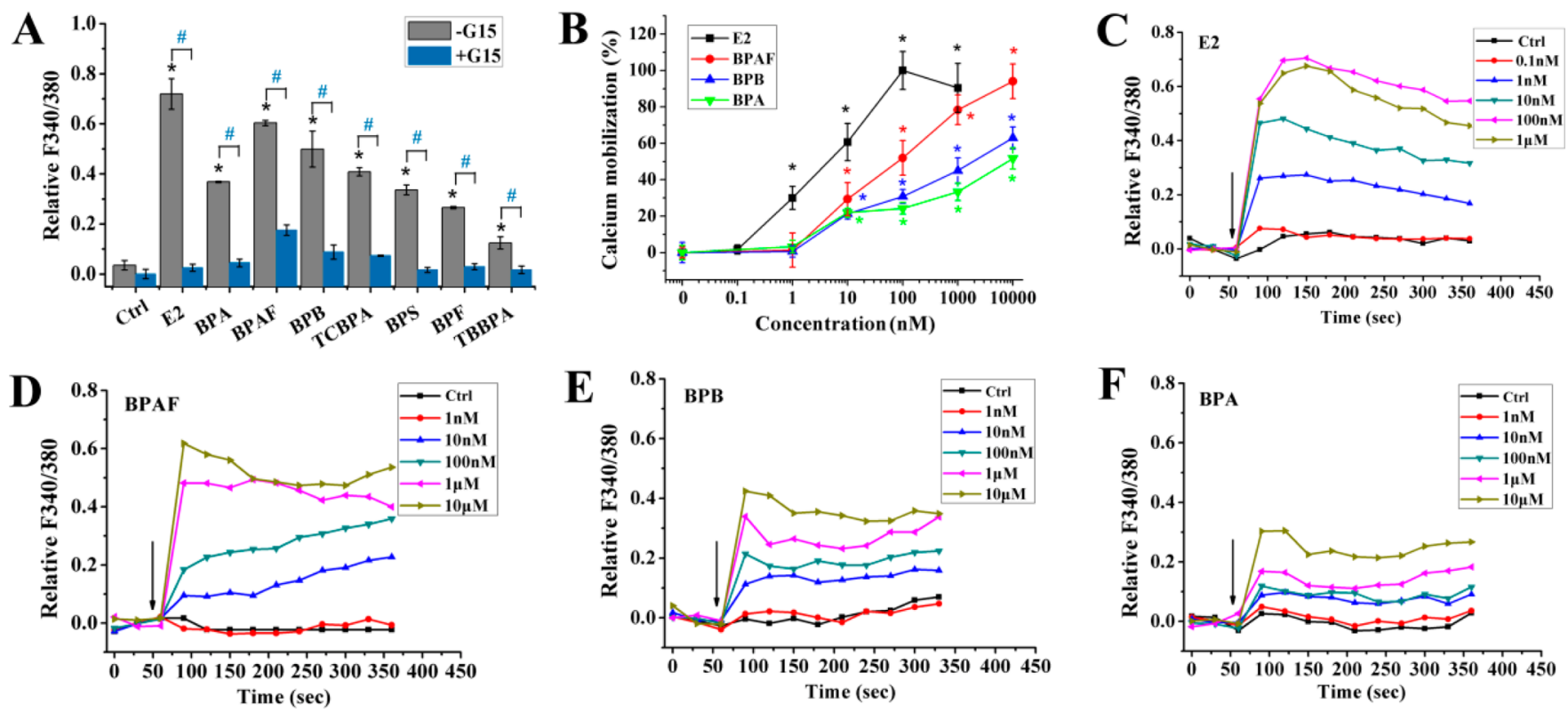

Figure 3. Effects of $\mathrm{E} 2$ and bisphenols on calcium mobilization in SKBR3 cells. (A) Calcium mobilization induced by $100 \mathrm{nM} \mathrm{E} 2$ and $1 \mu \mathrm{M}$ bisphenols in the absence or presence of $10 \mu \mathrm{M}$ G15. (B) Calcium mobilization induced by different concentrations of E2, BPAF, BPB, and BPA. The calcium mobilization was determined by setting the maximum effect induced by E2 as $100 \%$. The concentration at $0 \mathrm{nM}$ means the vehicle Ctrl group. (C-F) Various concentrations of E2, BPAF, BPB, and BPA stimulated the calcium mobilization in a time-dependent manner. The arrows indicate the time to add the compounds. ${ }^{*} p<0.05$ were compared with the Ctrl. $\# p<0.05$ compared with the groups treated with compounds in absence of G15.
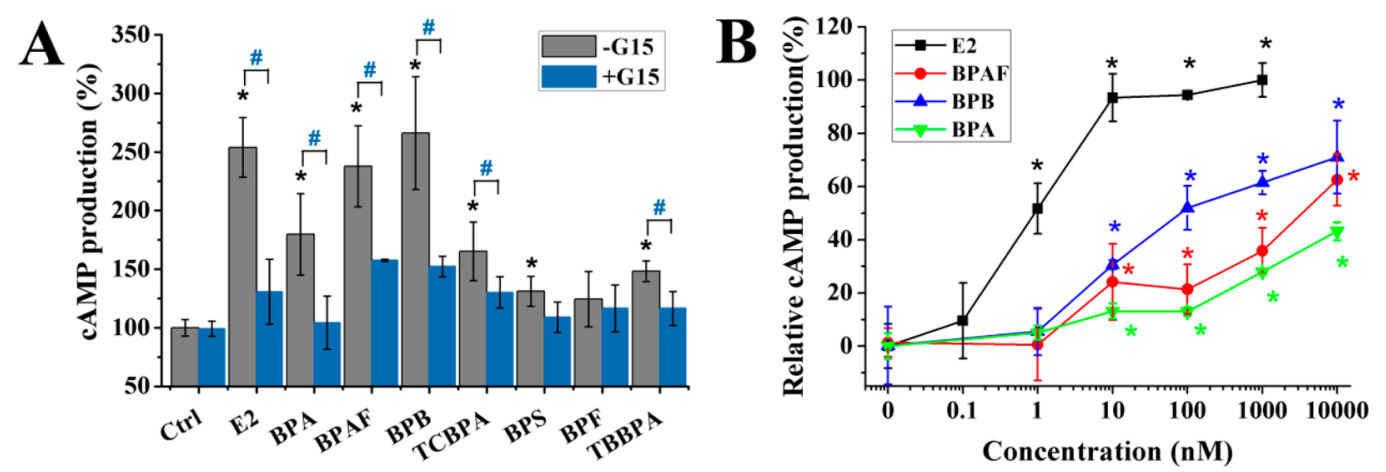

Figure 4. Effects of $\mathrm{E} 2$ and bisphenols on cAMP accumulation in SKBR3 cells. (A) The cAMP production induced by $100 \mathrm{nM}$ E2 and $1 \mu \mathrm{M}$ bisphenols in the absence or presence of $10 \mu \mathrm{M}$ G15. (B) The cAMP production induced by different concentrations of E2, BPAF, BPB, and BPA. The relative cAMP production was determined by setting the maximum effect induced by E2 as $100 \%$. The concentration at $0 \mathrm{nM}$ means the vehicle Ctrl group. ${ }^{*} p<0.05$ were compared with the Ctrl. $\# p<0.05$ compared with the groups treated with compounds in absence of G15.

Among the six tested BPA analogues, we found $1 \mu \mathrm{M}$ BPAF and $\mathrm{BPB}$ even showed higher potency in activating GPER subsequent signaling pathways than $1 \mu \mathrm{M}$ BPA (Figure $3 \mathrm{~A}$ and Figure 4A). These results suggest BPAF and BPB might have stronger GPER agonistic activity than BPA. To confirm this, we investigated the concentration-effect relationship of BPAF, $\mathrm{BPB}$, and BPA. At the same time, E2 was also tested as a positive control. As expected, E2 induced both the calcium mobilization and cAMP production in a concentrationdependent manner with the lowest effective concentration (LOEC) of $1.0 \mathrm{nM}$, and with an $\mathrm{EC}_{50}$ (the concentration needed to reach $50 \%$ of the maximum effect of E2) of $4.5 \mathrm{nM}$ and $1.0 \mathrm{nM}$ respectively (Figure $3 \mathrm{~B}$ and $4 \mathrm{~B}$ ). For BPAF, BPB and $\mathrm{BPA}$, they also stimulated the increase of calcium mobilization and cAMP production in a concentrationdependent manner with the LOEC of $10.0 \mathrm{nM}$ (Figure 3B$\mathrm{E}$ and Figure $4 \mathrm{~B})$. The $\mathrm{EC}_{50}$ values of calcium mobilization for $\mathrm{BPAF}, \mathrm{BPB}$, and $\mathrm{BPA}$ were $94.0 \mathrm{nM}, 1.7 \mu \mathrm{M}$, and $7.5 \mu \mathrm{M}$ respectively (Figure $3 \mathrm{~B}$ ). Compared to $\mathrm{BPA}, \mathrm{BPAF}$, and $\mathrm{BPB}$ presented much lower $\mathrm{EC}_{50}$ values, suggesting they had stronger agonistic activity than BPA. Similar results were found in the cAMP production assay. The $\mathrm{EC}_{50}$ values of cAMP production for BPAF, BPB, and BPA were about $3.3 \mu \mathrm{M}, 97.5$ $\mathrm{nM}$, and $>10.0 \mu \mathrm{M}$ respectively (Figure $4 \mathrm{~B}$ ). The above results demonstrated BPAF and BPB had stronger GPER agonistic activity than BPA, which is in accordance with the result that they had higher GPER binding affinity than BPA. The accordance suggests that the agonistic activity of a bisphenol to GPER might be dictated by its direct binding affinity with GPER.

In order to understand the agonistic mechanism of bisphenols on GPER, we studied their interactions with GPER by molecular docking simulation. According to the docking results from previous studies ${ }^{30,31}$ and our previous studies (unpublished data), the hydrogen bond with Asn 276 on transmembrane helix 6 (TM6) probably plays a key role in the 

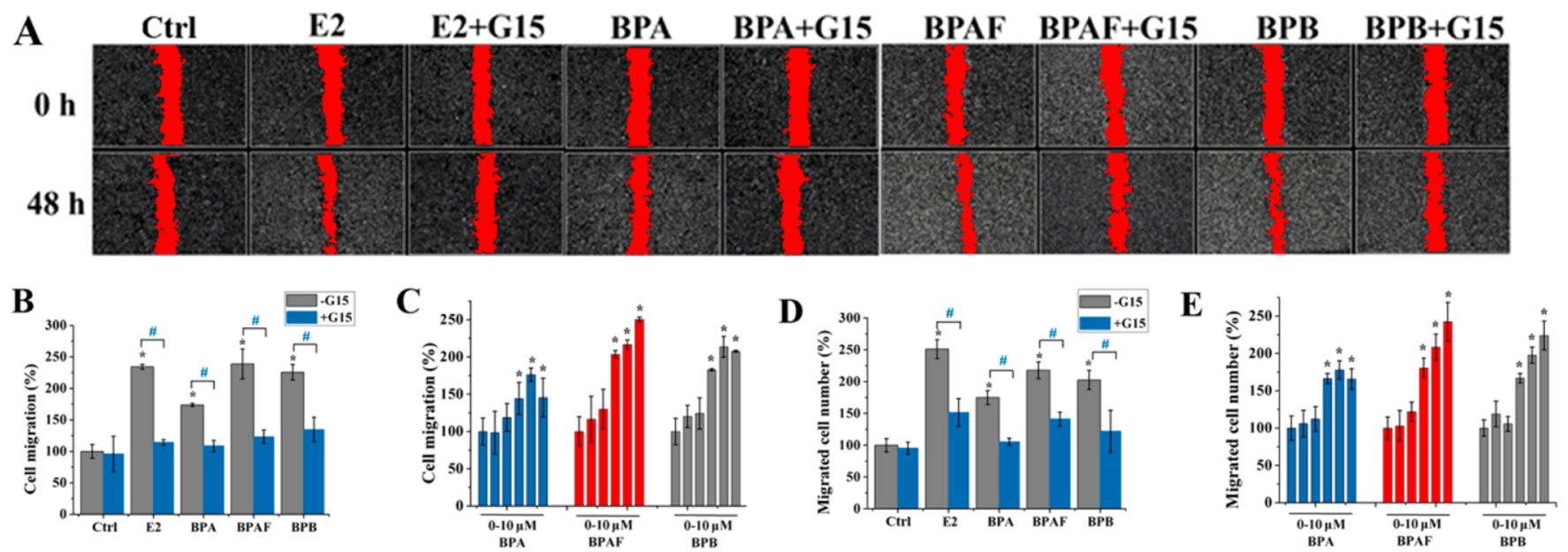

Figure 5. Effects of E2 and bisphenols on SKBR3 cells migration by wound-healing and Boyden chamber assays. (A) SKBR3 cells migration induced by $100 \mathrm{nM} \mathrm{E} 2$ and $1 \mu \mathrm{M}$ bisphenols in the absence or presence of $10 \mu \mathrm{M}$ G15 detected by wound-healing assay. The unhealed wound areas are highlighted in red. (B) The quantification of corresponding SKBR3 cells migration with treatment as indicated in section A. (C) SKBR3 cells migration induced by different concentrations of bisphenols $(0,1 \mathrm{nM}, 10 \mathrm{nM}, 100 \mathrm{nM}, 1 \mu \mathrm{M}$, and $10 \mu \mathrm{M})$ detected by wound-healing assays. (D) SKBR3 cells migration induced by $100 \mathrm{nM} \mathrm{E} 2$ and $1 \mu \mathrm{M}$ bisphenols in the absence or presence of $10 \mu \mathrm{M}$ G15 detected by Boyden chamber assay. (E) SKBR3 cells migration induced by different concentrations of bisphenols $(0,1 \mathrm{nM}, 10 \mathrm{nM}, 100 \mathrm{nM}, 1 \mu \mathrm{M}$, and $10 \mu \mathrm{M})$ detected by Boyden chamber assay. For wound-healing assay, the percentage of cell migration was calculated by the change of the wound area (area at $0 \mathrm{~h}$ minus area at $48 \mathrm{~h}$ ) of the treated group relative to the Ctrl group (100\%). For Boyden chamber method, the percentage of the migrated cell number was calculated by setting the migrated cells induced by Ctrl group as $100 \%$. ${ }^{*} p<0.05$ were compared with the Ctrl. \#p $<0.05$ compared with the groups treated with compounds in absence of G15.

activation of GPER by E2. However, we found the bisphenols did not form any hydrogen bond with Asn276. Alternatively, they formed hydrogen bonds with residue Gln138 on TM3, Phe206, and Cys207 on extracellular loop 2 (Figure 2B-D and SI Figure S6). Therefore, we speculated the agonistic mechanism of bisphenols on GPER might be different from E2. However, the exact agonistic mechanism of the bisphenols needs further investigations.

BPAF and BPB had Stronger Activity in Promoting GPER Mediated SKBR3 Cell Migration Than BPA. Previous studies have showed BPA could promote SKBR3 cell migration, a cell function mediated by the GPER pathway. ${ }^{12,13,29}$ Here, we wondered whether BPAF and BPB, which had higher binding affinity and agonistic activity to GPER, would also have higher effects on GPER mediated cell functions than BPA. Therefore, wound-healing and Boyden chamber assays were used to detect their effects on GPER mediated SKBR3 cell migration. For wound-healing assay, $1 \mu \mathrm{M}$ BPA, BPAF, and BPB induced the cell migration significantly and the effects were mostly eliminated by $10 \mu \mathrm{M}$ G15 (Figure 5A and B). These results suggest the three bisphenols induced the SKBR3 cell migration by activating the GPER pathway. As shown in Figure 5C, BPA, $\mathrm{BPAF}$, and $\mathrm{BPB}$ induced the cell migration in a concentrationdependent manner with the LOEC of $100 \mathrm{nM}$. Compared to $\mathrm{BPA}, \mathrm{BPAF}$, and BPB induced more promotion effects on the cell migration at the concentrations of $100 \mathrm{nM}, 1 \mu \mathrm{M}$, and 10 $\mu \mathrm{M}(p<0.05)$ (Figure 5C). Similar results were obtained by Boyden chamber assay. As shown in Figure 5D and E, BPAF and $\mathrm{BPB}$ presented more promotion effects on the cell migration than BPA at concentrations of $1 \mu \mathrm{M}$ and $10 \mu \mathrm{M}$ $(p<0.05)$. The above results demonstrated that BPAF and $\mathrm{BPB}$ had stronger activity in promoting SKBR3 cell migration via GPER pathway, which is in accordance with the result that BPAF and BPB had higher binding affinity and agonistic activity to GPER compared to BPA. Taken together, our study suggests strongly that, similar to BPA, BPA analogues could also act as agonists to activate GPER pathway, and lead to disruption of the GPER mediated physiological functions.

Toxicological Implication of BPA Analogues Based on Their Effects Through GPER Pathway. Previous investigations have showed BPA could exert estrogenic effects in animals at low doses. ${ }^{32,33}$ However, studies of the nuclear transcriptional regulatory activity of BPA have mostly been unable to explain the action of BPA in mediating the low-dose effects. Indeed, there is now strong evidence indicating that GPER pathway plays an important role in BPA's estrogenic effects at the low concentrations. ${ }^{15-17,34}$ This might be due to the fact that BPA has higher binding affinity with GPER (RBA of $1.1-2.8 \%$ compared to $\mathrm{E} 2{ }^{18}$ ) than with ER (RBA of $0.01-$ $0.1 \%$ compared to $\mathrm{E} 2^{35-37}$ ). Therefore, it could be inferred that BPA would act preferably on GPER but not ER at low concentrations. As shown in Table 1, by comparing the RBA values of BPA analogues with GPER obtained in our study and with ER obtained from literatures, ${ }^{35-38}$ it is obvious that they also have higher RBA values with GPER than ER (approximately 10-100-folds). These results indicate that the GPER pathway may also play important roles in the estrogenic effects of BPA analogues.

Many BPA analogues were designed and synthesized as candidates of BPA substitute with the expectation that they might have lower estrogenic disruption effects than BPA. Here, it deserves to be noted that two emerging BPA analogues, $\mathrm{BPAF}$ and $\mathrm{BPB}$, even had higher binding affinity and agonistic activity to GPER than BPA. Additionally, previous studies suggested that BPAF and BPB also had higher binding affinity and activity to ER than BPA. ${ }^{35,37,39}$ Based on our results for GPER as well as those of others for ER, BPAF, and BPB might have stronger estrogenic effects than BPA, thus might not be safe substitutes for BPA.

At present, the occurrence of BPAF and $\mathrm{BPB}$ in environmental compartments, foods or food containers and consumer products has been documented. ${ }^{7,9}$ Biomonitoring of the general 
population in various countries has also shown the presence of BPAF and BPB in humans. ${ }^{7}$ For example, a study reported the presence of $\mathrm{BPB}$ in human serum from endometriotic women in Italy at a mean concentration of $5.15 \mathrm{ng} / \mathrm{mL}(\sim 21 \mathrm{nM})$, even greater than BPA concentration (mean: $2.91 \mathrm{ng} / \mathrm{mL}, \sim 12$ $\mathrm{nM}) .{ }^{40}$ Based on our study, we found BPAF and BPB could disrupt the GPER pathway at nanomolar levels, triggering the subsequent GPER signaling pathway at $10 \mathrm{nM}$ and inducing SKBR3 cell migration at $100 \mathrm{nM}$. It is notable that the reported BPB serum concentration $(\sim 21 \mathrm{nM})$ of Italian endometriotic women is even higher than the LOEC for activating the GPER pathway detected in our experiments. Therefore, among the BPA analogues tested, we think BPAF and BPB are two emerging bisphenols which deserve more concerns in the future.

In the present study, we demonstrated for the first time that some BPA analogues bound to human GPER directly, with $\mathrm{BPAF}$ and $\mathrm{BPB}$ showing much higher affinity than BPA. Molecular docking simulation showed all the BPA analogues could fit into the GPER ligand binding pocket. We further demonstrated these BPA analogues had agonistic activity to GPER. BPAF and BPB may be able to disrupt the GPER related physiological functions at nanomolar concentrations. Our study suggests that the disruption of GPER pathway is one of the possible mechanisms for BPA analogues to exhibit estrogenic effects. Lastly, we found BPAF and BPB even had higher effects on GPER pathway than BPA, thus they might not be suitable as substitutes for BPA and deserve further studies for risk assessment.

\section{ASSOCIATED CONTENT}

\section{S Supporting Information}

The Supporting Information is available free of charge on the ACS Publications website at DOI: 10.1021/acs.est.7b03336.

Structure of the probe E2-F and tested compounds, fluorescence competitive binding method, Boyden chamber assay, wound-healing assay, homology modeling and molecular docking (PDF)

\section{AUTHOR INFORMATION}

\section{Corresponding Authors}

*(L.-H.G.) Phone/fax: 86010 62849685; e-mail: LHGuo@ rcees.ac.cn.

*(X.-M.R.) E-mail: xmren@rcees.ac.cn.

ORCID

Liang-Hong Guo: 0000-0003-1399-5716

Notes

The authors declare no competing financial interest.

\section{ACKNOWLEDGMENTS}

This work was supported by the Chinese Academy of Sciences (XDB14040100) and the National Natural Science Foundation of China (21407168, 21621064, 21375143, 91543203, 21577163, and 21477146).

\section{REFERENCES}

(1) Corrales, J.; Kristofco, L. A.; Steele, W. B.; Yates, B. S.; Breed, C. S.; Williams, E. S.; Brooks, B. W. Global assessment of bisphenol A in the environment: review and analysis of its occurrence and bioaccumulation. Dose-Response 2015, 13, 1-29.

(2) Chevalier, N.; Fenichel, P. Bisphenol A: targeting metabolic tissues. Rev. Endocr. Metab. Disord. 2015, 16, 299-309.
(3) Seachrist, D. D.; Bonk, K. W.; Ho, S. M.; Prins, G. S.; Soto, A. M.; Keri, R. A. A review of the carcinogenic potential of bisphenol A. Reprod. Toxicol. 2016, 59, 167-182.

(4) Ranciere, F.; Lyons, J. G.; Loh, V. H.; Botton, J.; Galloway, T.; Wang, T.; Shaw, J. E.; Magliano, D. J. Bisphenol A and the risk of cardiometabolic disorders: a systematic review with meta-analysis of the epidemiological evidence. Environ. Health 2015, 14, 46-69.

(5) Rochester, J. R. Bisphenol A and human health: a review of the literature. Reprod. Toxicol. 2013, 42, 132-155.

(6) Vandenberg, L. N.; Ehrlich, S.; Belcher, S. M.; Ben-Jonathan, N.; Dolinoy, D. C.; Hugo, E. R.; Hunt, P. A.; Newbold, R. R.; Rubin, B. S.; Saili, K. S.; Soto, A. M.; Wang, H.-S.; vom Saal, F. S. Low dose effects of bisphenol A. Endocr. Disruptors 2013, 1, 25078-25098.

(7) Chen, D.; Kannan, K.; Tan, H. L.; Zheng, Z. G.; Feng, Y. L.; Wu, Y.; Widelka, M. Bisphenol analogues other than BPA: environmental occurrence, human exposure, and toxicity-a review. Environ. Sci. Technol. 2016, 50, 5438-5453.

(8) Rochester, J. R.; Bolden, A. L. Bisphenol S and F: a systematic review and comparison of the hormonal activity of bisphenol A substitutes. Environ. Health Perspect. 2015, 123, 643-650.

(9) Caballero-Casero, N.; Lunar, L.; Rubio, S. Analytical methods for the determination of mixtures of bisphenols and derivatives in human and environmental exposure sources and biological fluids. a review. Anal. Chim. Acta 2016, 908, 22-53.

(10) Acconcia, F.; Pallottini, V.; Marino, M. Molecular mechanisms of action of BPA. Dose-Response 2015, 13, 1-9.

(11) Welshons, W. V.; Nagel, S. C.; Saal, F. S. V. Large effects from small exposures. III. Endocrine mechanisms mediating effects of bisphenol A at levels of human exposure. Endocrinology 2006, 147, $56-69$.

(12) Castillo Sanchez, R.; Gomez, R.; Perez Salazar, E. Bisphenol A induces migration through a GPER-, FAK-, Src-, and ERK2-dependent pathway in MDA-MB-231 breast cancer cells. Chem. Res. Toxicol. 2016, 29, 285-295.

(13) Zhang, K. S.; Chen, H. Q.; Chen, Y. S.; Qiu, K. F.; Zheng, X. B.; Li, G. C.; Yang, H. D.; Wen, C. J. Bisphenol A stimulates human lung cancer cell migration via upregulation of matrix metalloproteinases by GPER/EGFR/ERK1/2 signal pathway. Biomed. Pharmacother. 2014, 68, 1037-1043.

(14) Dong, S.; Terasaka, S.; Kiyama, R. Bisphenol A induces a rapid activation of ERK1/2 through GPR30 in human breast cancer cells. Environ. Pollut. 2011, 159, 212-218.

(15) Sheng, Z. G.; Zhu, B. Z. Low concentrations of bisphenol A induce mouse spermatogonial cell proliferation by $G$ protein-coupled receptor 30 and estrogen receptor-alpha. Environ. Health Perspect. 2011, 119, 1775-1780.

(16) Bouskine, A.; Nebout, M.; Bruecker-Davis, F.; Benahmed, M.; Fenichel, P. Low doses of bisphenol A promote human seminoma cell proliferation by activating PKA and PKG via a membrane G proteincoupled estrogen receptor. Environ. Health Perspect. 2009, 117, 10531058.

(17) Chevalier, N.; Bouskine, A.; Fenichel, P. Bisphenol A promotes testicular seminoma cell proliferation through GPER/GPR30. Int. J. Cancer 2012, 130, 241-242.

(18) Thomas, P.; Dong, J. Binding and activation of the seventransmembrane estrogen receptor GPR30 by environmental estrogens: a potential novel mechanism of endocrine disruption. J. Steroid Biochem. Mol. Biol. 2006, 102, 175-179.

(19) Fitzgerald, A. C.; Peyton, C.; Dong, J.; Thomas, P. Bisphenol A and related alkylphenols exert nongenomic estrogenic actions through a G protein-coupled estrogen receptor 1 (GPER)/epidermal growth factor receptor (EGFR) pathway to inhibit meiotic maturation of zebrafish oocytes. Biol. Reprod. 2015, 93, 135-146.

(20) Yamasaki, K.; Takeyoshi, M.; Yakabe, Y.; Sawaki, M.; Takatsuki, M. Comparison of the reporter gene assay for ER-alpha antagonists with the immature rat uterotrophic assay of 10 chemicals. Toxicol. Lett. 2003, 142, 119-131.

(21) Song, M.; Liang, D.; Liang, Y.; Chen, M.; Wang, F.; Wang, H.; Jiang, G. Assessing developmental toxicity and estrogenic activity of 
halogenated bisphenol A on zebrafish (Danio rerio). Chemosphere 2014, 112, 275-281.

(22) Cano-Nicolau, J.; Vaillant, C.; Pellegrini, E.; Charlier, T. D.; Kah, O.; Coumailleau, P. Estrogenic effects of several BPA analogs in the developing zebrafish brain. Front. Neurosci. 2016, 10, 112-126.

(23) Kitamura, S.; Suzuki, T.; Sanoh, S.; Kohta, R.; Jinno, N.; Sugihara, K.; Yoshihara, S.; Fujimoto, N.; Watanabe, H.; Ohta, S. Comparative study of the endocrine-disrupting activity of bisphenol A and 19 related compounds. Toxicol. Sci. 2005, 84, 249-259.

(24) Ruan, T.; Liang, D.; Song, S.; Song, M.; Wang, H.; Jiang, G. Evaluation of the in vitro estrogenicity of emerging bisphenol analogs and their respective estrogenic contributions in municipal sewage sludge in China. Chemosphere 2015, 124, 150-155.

(25) Li, M.; Guo, J.; Gao, W. H.; Yu, J. L.; Han, X. Y.; Zhang, J.; Shao, B. Bisphenol AF-induced endogenous transcription is mediated by ER alpha and ERK1/2 activation in human breast cancer cells. PLoS One 2014, 9, 94725-94733.

(26) Vinas, R; Watson, C. S. Bisphenol S disrupts estradiol-induced nongenomic signaling in a rat pituitary cell line: effects on cell functions. Environ. Health Perspect. 2013, 121, 352-358.

(27) Revankar, C. M.; Cimino, D. F.; Sklar, L. A.; Arterburn, J. B.; Prossnitz, E. R. A transmembrane intracellular estrogen receptor mediates rapid cell signaling. Science 2005, 307, 1625-1630.

(28) Bologa, C. G.; Revankar, C. M.; Young, S. M.; Edwards, B. S.; Arterburn, J. B.; Kiselyov, A. S.; Parker, M. A.; Tkachenko, S. E.; Savchuck, N. P.; Sklar, L. A.; Oprea, T. I.; Prossnitz, E. R. Virtual and biomolecular screening converge on a selective agonist for GPR30. Nat. Chem. Biol. 2006, 2, 207-212.

(29) Pupo, M.; Pisano, A.; Lappano, R.; Santolla, M. F.; De Francesco, E. M.; Abonante, S.; Rosano, C.; Maggiolini, M. Bisphenol $A$ induces gene expression changes and proliferative effects through GPER in breast cancer cells and cancer-associated fibroblasts. Environ. Health Perspect. 2012, 120, 1177-1182.

(30) Arnatt, C. K.; Zhang, Y. G protein-coupled estrogen receptor (GPER) agonist dual binding mode analyses toward understanding of its activation mechanism: a comparative homology modeling approach. Mol. Inf. 2013, 32, 647-658.

(31) Mendez-Luna, D.; Martinez-Archundia, M.; Maroun, R. C.; Ceballos-Reyes, G.; Fragoso-Vazquez, M. J.; Gonzalez-Juarez, D. E.; Correa-Basurto, J. Deciphering the GPER/GPR30-agonist and antagonists interactions using molecular modeling studies, molecular dynamics, and docking simulations. J. Biomol. Struct. Dyn. 2015, 33, 2161-2172.

(32) vom Saal, F. S.; Welshons, W. V. Large effects from small exposures. II. The importance of positive controls in low-dose research on bisphenol A. Environ. Res. 2006, 100, 50-76.

(33) vom Saal, F. S.; Hughes, C. An extensive new literature concerning low-dose effects of bisphenol A shows the need for a new risk assessment. Environ. Health Perspect. 2005, 113, 926-33.

(34) Ge, L. C.; Chen, Z. J.; Liu, H. Y.; Zhang, K. S.; Liu, H.; Huang, H. B.; Zhang, G.; Wong, C. K.; Giesy, J. P.; Du, J.; Wang, H. S. Involvement of activating ERK1/2 through $G$ protein coupled receptor 30 and estrogen receptor alpha/beta in low doses of bisphenol A promoting growth of sertoli TM4 cells. Toxicol. Lett. 2014, 226, 81-89.

(35) Delfosse, V.; Grimaldi, M.; Pons, J. L.; Boulahtouf, A.; le Maire, A.; Cavailles, V.; Labesse, G.; Bourguet, W.; Balaguer, P. Structural and mechanistic insights into bisphenols action provide guidelines for risk assessment and discovery of bisphenol A substitutes. Proc. Natl. Acad. Sci. U. S. A. 2012, 109, 14930-14935.

(36) Blair, R. M.; Fang, H.; Branham, W. S.; Hass, B. S.; Dial, S. L.; Moland, C. L.; Tong, W. D.; Shi, L. M.; Perkins, R.; Sheehan, D. M. The estrogen receptor relative binding affinities of 188 natural and xenochemicals: structural diversity of ligands. Toxicol. Sci. 2000, 54, $138-153$.

(37) Okada, H.; Tokunaga, T.; Liu, X.; Takayanagi, S.; Matsushima, A.; Shimohigashi, Y. Direct evidence revealing structural elements essential for the high binding ability of bisphenol A to human estrogen-related receptor-gamma. Environ. Health Perspect. 2008, 116, $32-38$.

(38) Lee, H. K.; Kim, T. S.; Kim, C. Y.; Kang, I. H.; Kim, M. G.; Jung, K. K.; Kim, H. S.; Han, S. Y.; Yoon, H. J.; Rhee, G. S. Evaluation of in vitro screening system for estrogenicity: comparison of stably transfected human estrogen receptor-alpha transcriptional activation (OECD TG455) assay and estrogen receptor (ER) binding assay. J. Toxicol. Sci. 2012, 37, 431-437.

(39) Matsushima, A.; Liu, X.; Okada, H.; Shimohigashi, M.; Shimohigashi, Y. Bisphenol AF is a full agonist for the estrogen receptor ER alpha but a highly specific antagonist for ER beta. Environ. Health Perspect. 2010, 118, 1267-1272.

(40) Cobellis, L.; Colacurci, N.; Trabucco, E.; Carpentiero, C.; Grumetto, L. Measurement of bisphenol A and bisphenol B levels in human blood sera from endometriotic women. Biomed. Chromatogr. 2009, 23, 1186-1190. 